\title{
The Use of Capillarity Surfaces to Predict Phase Distributions In Mixed-Wet Porous Media.
}

\author{
MCDOUGALL, Steven R, Heriot-Watt University Edinburgh, UK \\ DIXIT, Ashok B, Heriot-Watt University Edinburgh, UK \\ SORBIE, Kenneth S, Heriot-Watt University Edinburgh, UK
}

Paper presented at the 5th European Conference on the Mathematics of Oil Recovery, Loeben, Austria, 3-6 Sept. 1996.

\begin{abstract}
Pore-scale network models have been used to study a range of nonuniformly-wet systems, their associated capillary pressure curves, imbibition characteristics and waterflood recovery efficiencies. Recent research has demonstrated that wettability alteration depends upon a variety of factors, including; ageing temperature, brine $\mathrm{pH} /$ composition, crude oil composition, and connate water saturation. The premise here, however, is that the net result of these parameters is to produce a particular distribution of contact angles within the pore system. Consequently, the ageing process characterising a particular wettability experiment produces oil-wet pores with effective contact angles in the range $\theta_{\mathrm{O}} 1<\theta_{\mathrm{O}}<\theta_{\mathrm{O} 2}$ and water-wet pores with $\theta_{w 1}<\theta_{w}<\theta_{w} 2$. The underlying pore-scale physics has been further explored by defining a capillarity surface which, when examined in conjunction with percolation theory, can be used to predict phase distributions for any contact angle range. Indeed, results show that a sample containing pores with a range of effective contact angles can imbibe up to $50 \%$ more water than one that is strongly water-wet. A number of apparently contradictory experimental observations have also been successfully reproduced and interpreted in light of this new theory.
\end{abstract}

\section{INTRODUCTION}

Numerous reports of experimental work relating to the role of non-uniform wettability in various aspects of oil recovery have been published, although a precise taxonomy of wettability is still lacking. Ageing temperature, brine pH/composition, crude oil composition, and connate water saturation have all been shown to play an important role in determining the ultimate wettability state of a system (Dubey and Waxman, 1989; Hirasaki et al., 1990; Dubey and Doe, 1991; Jadhunandan and Morrow, 1991; Wolcott et al., 1991, 1993; Buckley, 1993; Kovscek et al, 1993). Unfortunately, variations in laboratory procedure and experimental materials make generalised conclusions almost impossible and the precise wettability conditions prevailing at the end of a conditioning procedure are frequently difficult to deduce. Moreover, there are even serious doubts concerning the cross-correlation of the two main experimental methods used to determine core wettability, i.e. Amott and USBM tests. Indeed, until some clear theoretical interpretation of such matters is available, it is difficult to see how results from different laboratories can be adequately reconciled and understood.

To this end, the work presented here focuses upon the systematic investigation of wettability alteration at the pore-scale and its subsequent impact upon waterflood displacement efficiency. Pore-scale models have been used to study a range of nonuniformly-wet systems, their associated capillary pressure curves, spontaneous water imbibition characteristics and waterflood recovery behaviour. The models are capable of simulating the full flooding cycle characteristic of laboratory wettability tests (primary drainage, "ageing", water imbibition, water drive, oil imbibition, oil drive).

The first part of the cycle (primary drainage of water by oil from a water-wet system) is followed by an "ageing" process, whereby wettability alterations occur at the pore-scale. As noted earlier, in a physical system, wettability alteration depends upon a variety of factors. The premise here, however, is that the net result of these parameters is to produce a particular distribution of contact angles within the system. Consequently, the simulated ageing process produces oil-wet pores with contact angles in the range $\theta_{\mathrm{o} 1}<\theta_{\mathrm{O}}<\theta_{\mathrm{o}} 2$ and water-wet pores with $\theta_{\mathrm{w} 1}<\theta_{\mathrm{w}}<\theta_{\mathrm{w} 2}$. Of course, the precise range operating during an experiment depends upon complex interactions amongst the factors mentioned above. Thus, instead of quantifying the effects of contact angle range for one particular case, the model has been used to examine the sensitivities of a cycle of displacements running under a number of different wettability scenarios. The results have been illuminating and have led to the concept of a capillarity surface, which can be used to predict phase distributions for any contact angle range. A number of contradictory experimental observations have been reproduced and interpreted using this new approach. 


\section{FLOW SIMULATIONS IN SYSTEMS OF NON-UNIFORM WETTABILITY}

\subsection{Generation of Model and the Flooding Cycle}

A 3-D pore-scale model of average co-ordination number four $(\mathrm{z}=4)$ has been used for the simulations. For simplicity, a uniform pore size distribution has been assumed: radii in the range of 0.1 to $100 \mu \mathrm{m}$ are assigned to pore elements by using a random number generator. All pores are assumed to be of equal length $(100 \mu \mathrm{m})$ and have a cylindrical geometry. Note, however, that these are not limitations of the model, as any other pore size distribution and/or volume function can be included in order to mimic alternative geometries. Such sensitivities have been carried out and overall trends are very similar to those reported later. The full five-flood cycle described above, including wettability alterations, is performed on all networks. The detailed algorithms corresponding to each stage of the cycle have been presented elsewhere (McDougall et al, 1995) and only the most important details are reported here.

The primary drainage simulation begins with a strongly water-wet network $\left(\theta=0^{\circ}\right.$ in all pores) at $100 \%$ water saturation. The capillary pressure is increased step by step from zero to a value at which the continuity of water is lost (hydraulic discontinuity). At each pressure step, water and oil saturations are evaluated and the primary drainage capillary pressure curve constructed.

The experimental work of various researchers indicate that components of the crude oil interact differently with different mineral substrates in the rock e.g. quartz, feldspars, clays, etc (Jordan et al, 1994). Therefore, we believe that the wettability alterations that have occurred in actual reservoirs, as well as those associated with core ageing experiments, are non-uniform; i.e. contact angles are not identical for all water-wet or oil-wet pores. These pore level wettability variations are incorporated in the network model as a result of ageing. It is assumed that the pores occupying initial water remain strongly water-wet $\left(\theta=0^{\circ}\right)$ as they are not in contact with oil. However, the effective contact angles of the pores occupying oil after primary drainage may change during the ageing process. In the present study, a mixed-wet model of non-uniform wettability is considered as being most realistic: this is a system where the largest pores containing oil after primary drainage preferentially become oil-wet (Figure 1). This assumption is rigorously true for cylindrical pore elements and a heuristic argument based upon surface asperities can be invoked to generalise the assumption to pores of random geometry. The argument is outlined in Figure 2.

In the oil-wet pores, effective contact angles are distributed randomly from 91 to $180^{\circ}$. Note that the remaining fraction of pores occupying oil after primary drainage remain water-wet (by definition, $\theta<90^{\circ}$ ) and therefore, contact angles are distributed randomly between 0 and $89^{\circ}$ in this region. We would like to emphasise two things: (i) no experimental techniques are currently available to measure such contact angle distributions in natural porous media and thus, the pore-scale simulator presented here provides an ideal theoretical tool to investigate and predict the expected consequences of various wettability/contact angle variations; (ii) the term "effective contact angle" is used here to include the effects of pore geometry - wall curvature and measured fluid/solid contact angles combine to give an effective meniscus curvature that we characterise using the effective contact angle).

The subsequent steps in the flooding cycle, namely water imbibition, forced water drive, oil imbibition and forced oil drive are simulated using this modified/aged network.

Many researchers have observed film-flow via thin wetting films in multiphase flow experiments using glass micromodels. In the present model, a realistic partial film-flow mechanism is implemented during all four stages of the cycle following ageing. Note, that film-flow affects not only the imbibition of a phase into the network but also its escape from it during other parts of the flooding cycle. The details of the partial film-flow mechanism are as follows:

Both water-wet and oil-wet pores are subdivided into two categories, depending upon their contact angle: (i) it is assumed that water can invade by film flow only in pores with contact angles in the range 0 to $\theta_{c w}$ (strongly water-wet), whilst oil can only escape through films in pores having contact angles between $\theta_{c o}$ and $180^{\circ}$ (strongly oil-wet). $\theta_{c w}$ and $\theta_{c o}$ are critical cut-off values; (ii) a continuous path of water-filled pores is essential for the remaining weakly water-wet pores $\left(\theta_{\mathrm{cw}}<\theta<89^{\circ}\right)$ to become available for invasion. Similarly, oil-wet pores with $91^{\circ}<\theta<\theta_{\text {co }}$ require a continuum of oil to the outlet of the system in order to escape. Analogous arguments hold when oil is the invading phase. In the present study, the values of $\theta_{\mathrm{cw}}$ and $\theta_{\mathrm{co}}$ have been taken as $45^{\circ}$ and $135^{\circ}$, respectively. The partial film-flow mechanism is considered by the authors to be the most realistic mechanism operating in natural porous media

It is well known that the advancing contact angle is usually higher than the receding contact angle (Morrow, 1975). This experimentally observed phenomenon is also incorporated in the model. A 
distribution of advancing contact angles is used during imbibition processes whereas receding contact angles are employed for drainage processes. In all the simulations presented here, the receding contact angle was taken to be $20 \%$ less than the corresponding advancing angle (broadly in line with experimental data).

\section{3 \\ RESULTS AND DISCUSSION}

The more "severe" the ageing conditions under which wettability alteration occurs, the greater the number of those pores occupied by oil after primary drainage that will become oil-wet. In the present study, a number of different ageing conditions are simulated simply by converting different fractions of oil-filled pores from a waterwet to an oil-wet state after the primary drainage simulation. Note that the pores occupied by initial water remain strongly water-wet $\left(\theta=0^{\circ}\right)$ even after ageing and therefore, for a finite initial water saturation, the fraction of oil-wet pores in the network $(\alpha)$ is always less than one. In the present study, primary drainage is terminated at water hydraulic discontinuity. According to percolation theory, in an infinite network of a given coordination number ( $\mathrm{z})$, hydraulic discontinuity would be observed when the fraction of wateroccupied pores in the network is slightly less than the corresponding percolation threshold $\left(\mathrm{P}_{\mathrm{T}}\right)$ of the system (Stauffer and Aharony, 1994). Therefore, for this study, the maximum value of $\alpha\left(\alpha_{\max }\right)$ is equal to $1-P_{\mathrm{T}}$. For $\mathrm{z}=4$, this corresponds to $\alpha \approx 0.625$. We note that it is possible to have, $\alpha_{\max }>1-\mathrm{P}_{\mathrm{T}}$, if we continue to allow water to escape via thin films along water-wet clusters after hydraulic discontinuity has been reached. This work is currently in progress.

Although a wide range of sensitivities has been performed during this work (using different film-flow mechanisms, different co-ordination numbers, mixedand fractionally-wet systems), discussion will be restricted here to the role played by distributed contact angles during oil/water displacements. Results from systems containing pores with distributed contact angles (i.e. water-wet pores with $0^{\circ}<\theta<90^{\circ}$ and oil-wet pores with $\left.90^{\circ}<\theta<180^{\circ}\right)$, will be compared with those from systems containing pores with single-valued contact angles (i.e. all water-wet pores having $\theta=0^{\circ}$ and all oil-wet pores having $\theta=180^{\circ}$, say).

\subsection{Capillary Pressure Curves}

Single-Valued Contact Angles: Simulated capillary pressure curves for a variety of networks with singlevalued contact angles are presented in Figure 3. Consider first the strongly water-wet network $(\alpha=0$, Figure 3a). Following the imbibition curve downwards from the end of primary drainage, it is found that about
$30 \%$ of the total pore volume is occupied by water after water imbibition. The third and fourth parts of the flooding cycle (forced water drive and oil imbibition), produce curves that are completely vertical: this is due to the absence of any oil-wet pores in the network The final stage of the procedure, oil-drive, is characterised by its hysteresis with the water imbibition curve.

An immediate observation from Figure 3 is that the amount of water imbibed decreases with an increase in $\alpha$. This is due to: (i) a reduction in the number of waterwet pores in the network, which cuts down the number of possible imbibition paths, and (ii) the connectivity of the system $(z=4)$, which means that the number of isolated water-wet clusters present after ageing also increases with $\alpha$. These isolated water-wet clusters are effectively shielded from the invading water by oil-wet pores during imbibition.

The water saturation at the end of water-drive is found to remain almost constant up to $\alpha=0.375$, because no connected path of oil-wet pores is yet available to transfer oil via film-flow. Only after $\alpha>0.375$ should any difference be seen, as this is the percolation threshold for oil-wet pores. This has been borne out by simulation for $\alpha=0.469$ and 0.625 , the water saturations after forced water drive are found to be much higher than those with $\alpha=0.156$ and 0.313 .

A continuous oil-wet cluster from the surface is also essential for oil imbibition during stage four of the cycle: no imbibition of oil is observed for $\alpha=0.156$ and 0.313 as expected. For $\alpha>0.375$, however, the extent of oil imbibition increases rapidly, as the oil-wet pores aggregate to form larger spanning clusters.

When $\alpha=0.625$ (i.e. when all oil-filled pores at the end of primary drainage have become oil-wet), no imbibition of water takes place at all because all of the water-wet pores are associated with disconnected irreducible water (Figure 3c): no oil drainage occurs for the same reason. Note also, that the end-of-cycle water saturation is slightly higher than the connate water saturation, which implies that water ultimately remains trapped in both oil-wet and water-wet pores. These observations are consistent with experimental results reported by Salathiel (1973).

Distributed Contact Angles: Having outlined the typical behaviour of single-valued contact angle systems, compare now the results obtained from systems with distributed contact angles. The corresponding capillary pressure curves are shown in Figure 4. The extent of water imbibition differs significantly from that obtained from single-valued contact angle systems. Compare the results for $\alpha=0$ (note that although $\alpha=0$ means that no oil-wet pores exist, weakly water-wet pores $-0^{\circ}<\theta<89^{\circ}$ - are present). The extent of spontaneous water imbibition increases by $\sim 55 \%$ to $0.384 \mathrm{PV}$ when the contact angles are distributed between $0^{\circ}$ and $89^{\circ}$. This compares with only $0.248 \mathrm{PV}$ imbibed with single-valued contact angles $\left(\theta=0^{\circ}\right)$. Moreover, for $\alpha<0.375$ (percolation threshold), the water saturations at the end of the forced water drive 
decrease significantly with $\alpha$ when contact angles are distributed. Remember that this effect was absent after waterflooding systems characterised by single-valued contact angles. These results clearly indicate that a distribution of contact angles at the pore-scale plays a crucial role in determining recovery in systems of nonuniform wettability. In fact, it will now be shown that it is the actual range of contact angles that is of primary importance.

Table 1 shows water saturations and \% OOIP (original oil in place) recovered after water imbibition for various effective contact angle ranges in a $100 \%$ water-wet system. Notice that the extent of water imbibition with distributed contact angles up to $30^{\circ}$ is almost identical with that resulting from single-valued contact angles. Even with $\theta=0-60^{\circ}$, the water saturation is only marginally higher than that obtained with all $\theta=0^{\circ}$. Only when contact angles are assigned from the range $0-89^{\circ}$ is water imbibition substantially enhanced - implying something special about the range $60-89^{\circ}$. This has been confirmed by distributing the contact angles within the restricted ranges $45-89^{\circ}$ and $60-89^{\circ}$ (see Table 1). This has major implications for laboratory wettability tests: these results show that a sample containing pores with a range of contact angles can imbibe up to 50\% more water than one that is strongly water-wet $\left(\theta=0^{\circ}\right)$. What is even more surprising, is that this is even true for a system that would appear to be neutrally-wet on the basis of contact angle measurements $\left(\theta=60-89^{\circ}\right)$. So, what are the reasons for this intriguing behaviour?

\subsection{Contact Angle Distributions and Capillarity Surfaces}

Consider the simple example of five oil-filled cylindrical capillary tubes of differing radii. When the oil/water contact angle is the same in each tube, the imbibition filling sequence is entirely governed by pore radius through the Young-Laplace equation - the smallest tube fills first, and so on (Figure 5a). With distributed contact angles, however, this need no longer be the case, as shown in Figure $5 \mathrm{~b}$; the filling sequence can be altered dramatically, because some of the smaller pores may now possess large contact angles and can only be filled at very low capillary pressures. This is rather intuitive and not too surprising, but the next example highlights a less-obvious effect. What happens to the filling sequence if the contact angle of each tube is simply halved; i.e. if the set of contact angles is changed from $\left\{9^{\circ}, 15^{\circ}, 50^{\circ}, 76^{\circ}, 88^{\circ}\right\}$ to $\left\{4.5^{\circ}, 7.5^{\circ}, 25^{\circ}, 38^{\circ}\right.$, $\left.44^{\circ}\right\}$ ? On the face of it, the filling sequence should be the same as before, as all angles have simply been reduced by a factor of 2 and their rank order remains the same. The new filling sequence, shown in Figure $6 c$, demonstrates that this need not be the case, however. This rather surprising result is due to the nonlinearity of the cosine function and its implicit nonlinear effect upon capillary pressure. The combined effects of contact angle and capillary entry radius can perhaps best be understood by defining a capillarity surface. This is a three-dimensional plot of capillary pressure as a function of both contact angle and capillary entry radius; i.e. the surface:

$$
P_{c}(r, \theta)=\frac{2 \sigma \cos \theta}{r}
$$

A typical example is shown in Figure 6a - the nonlinearity is self-evident. An important consequence of such a plot is that approximate phase distributions can be predicted for any array of capillary elements (including interconnected networks) simply by examining the associated surface in the light of percolation theory. This is best illustrated with reference to a fully connected 3D water-wet network $(\mathrm{z}=6)$, which remains free of additional topological complications.

By taking a number of sequential horizontal crosssections through the capillarity surface (i.e. planes of constant Pc), the progress of the imbibition floods can be studied as capillary pressure decreases (note that drainage floods can also be analysed, but accessibility has to be taken into account). Pores lying on the flat plateau of the sliced surface must have capillary entry pressures greater than that corresponding to the horizontal cut-off value and, if accessible, must be filled with water. Those pores not lying on the flat plateau must therefore be filled with oil as shown in Figure 6b. Capillarity surfaces for any range of contact angles or capillary entry radii can easily be plotted and sensitivities analysed. Such an analysis will now be conducted and used to examine two issues arising from the simulation results described earlier.

The first issue relates to the effect of contact angle range upon the early stages of water imbibition. To investigate this, four ranges are considered and a constant capillary pressure cut-off is used to slice all four capillarity surfaces at the same point during the displacement. The results are given in Figure 7. They clearly show that the amount of water initially imbibed reduces considerably once the contact angle range incorporates weakly waterwet pores $\left(60^{\circ}<\theta<90^{\circ}\right)$.

The second, more important, issue arising from the simulation studies, concerns the reduction seen in residual oil once weakly water-wet pores are incorporated into the system. By examining the capillarity surface in light of percolation theory, the reasons for this behaviour become clear. For a wellconnected network with $\mathrm{z}=6$, the percolation threshold is approximately 0.25 (Stauffer and Aharony, 1994), so at the end of water imbibition, about $75 \%$ of the pores will contain water. Hence, the residual oil saturation can be estimated for all four cases by slicing the corresponding capillarity surface at a capillary pressure value that leaves $75 \%$ of the pores lying in the plateau region. The unshaded regions in Figure 8 therefore show the distributions of oil-filled pores at the end of water imbibition. Although the unshaded regions all have the same area (approximately $25 \%$ of the square), their shapes differ: it can therefore be concluded from the figure that residual oil should be maximum when the contact angles are all $0^{\circ}$ and minimum when they range from $0^{\circ}$ to $90^{\circ}$. 
The two sets of sensitivities just described mean that the capillary pressure curves from the four systems must actually cross one another. The implication for petrophysical practice is that wettability-imbibition trends depend entirely upon the capillary pressure cutoff employed in the laboratory. Moreover, the results presented here demonstrate that the contact angle range plays a major role in determining the form of capillary pressure curves and residual saturations.

\subsection{Oil Production During Water Imbibition}

The water imbibition capillary pressure curves as functions of produced OOIP from single-valued and distributed contact angle networks are given in Figure 9. The imbibition curve for the single-valued water-wet system $\left(\theta=0^{\circ}\right.$ for all pores in the network) is also given in Figure $9 \mathrm{~b}$ for reference. With single-valued contact angles, imbibition is maximum in a $100 \%$ water-wet system $(\alpha=0)$ and diminishes monotonically with an increase in the fraction of oil-wet pores. In the case of distributed contact angles, however, oil production for $\alpha=0$ and $\alpha=0.156$ is actually higher than that obtained from the strongly water-wet system. For larger $\alpha$, production again decreases.

These results, when viewed in light of the earlier theoretical discussion, may go some way towards a clearer interpretation of many contradictory experimental results of the past. Jadhunandan (1990) has reported similar experimental trends from cores aged using various brines at $26^{\circ} \mathrm{C}$. For a brine composition of $4 \% \mathrm{NaCl}$ and $0.2 \% \mathrm{CaCl}_{2}$, for example, the oil produced by water imbibition was found to be higher than that observed in a strongly water-wet core: no explanation was given. The work reported here would suggest that the increase in water imbibition in the aged core is probably due to a distribution of contact angles at the pore-scale; such anomalous behaviour cannot be explained by assuming single valued contact angles. Other experimental examples (Graue et al, 1994, Ma et al, 1994) are also consistent with the theory developed here. Such experimental evidences provide useful support for the physics underpinning the pore-scale simulator. A great deal of additional experimental data has been interpreted using a regime-based framework based upon the network model described here. Details can be found in Dixit et al (1996).

\section{CONCLUSIONS}

Capillary pressure curves from mixed-wet systems have been analysed using a 3-D pore-scale simulator which takes into account many pore level flow processes: nonuniform wettability alterations, partial film flow, trapping of wetting and non-wetting phases, variations in advancing and receding contact angles, etc.

It has been proposed that the ageing of cores produces pores with a distribution of effective contact angles; i.e. oil-wet pores in the range $\theta_{\mathrm{o} 1}<\theta_{\mathrm{o}}<\theta_{\mathrm{o} 2}$ and water-wet pores with $\theta_{\mathrm{W} 1}<\theta_{\mathrm{W}}<\theta_{\mathrm{W} 2}$.
Simulations predict that final waterflood recoveries from weakly water-wet (or even weakly oil-wet) systems can often exceed those from strongly water-wet systems (even though initial imbibition rates may lead to the opposite conclusion).

The underlying pore level physics has been explored by defining a capillarity surface which incorporates the combined effects of both contact angle and pore dimension during the imbibition process.

Theoretical models derived in this paper, in conjunction with pore-scale simulation, have led to the development of a new analytical framework within which apparently contradictory wettability experiments can be reconciled.

\section{NOMENCLATURE}

$\mathrm{P}_{\mathrm{T}}=$ Percolation threshold

Swi $=$ Initial water saturation

$z=$ Mean co-ordination number

$\alpha=$ Fraction of oil-wet pores in the network

$\alpha_{\mathrm{cr}}=$ Critical fraction of oil-wet pores

\section{ACKNOWLEDGEMENTS}

The authors would like to express their gratitude to the following organisations for their invaluable financial support: BP Exploration Ltd., the UK Department of Trade and Industry, Exxon Production Research Co., Shell UK Ltd., and Statoil. Useful discussions with Martin Blunt, Chris Brown, Pete Salino, Dave Stern, Paal-Eric Øren and Jill Buckley are also gratefully acknowledged.

\section{REFERENCES}

Buckley, J.S. "Asphaltene Precipitation and Crude Oil Wetting", Paper SPE 26675 presented in 68th Annual Technical Conference and Exhibition, Oct. 3-6 (1993), Houston.

Dixit, A B, McDougall, S R, Sorbie, K S, and Buckley, J $S$, "Pore Scale Modelling of Wettability Effects and their Influence on Oil Recovery", SPE 35451 presented at the 1996 SPE/DOE Tenth Symposium on Improved Oil Recovery, Tulsa, OK, 211-24 April.

Dubey, S.T. and Waxman, M.H., "Asphaltene Adsorption and Desorption From Mineral Surface", Paper SPE 18462 presented at SPE International Symposium on Oil field Chemistry, Feb. 9-10 (1989), Houston.

Dubey, S.T. and Doe, P.H.," Base Number and Wetting Properties of Crude Oils" Paper SPE 22598 presented in 66th Annual Technical Conference and Exhibition, Oct. 6-9 (1991), Dallas.

Graue, A., Tonheim, E. and Baldwin, B., " Control and Alteration of Wettability in Low-Permeability Chalk", 
Paper presented at 1994 International Symposium on Evaluation Reservoir Wettability and Its Effect on Oil Recovery, Laramie, Sept. 21-23.

Hirasaki, G.J., Rohan, J.A., Dubey, S.T. and Niko, H., "Wettability Evaluation During Restored-State Core Analysis" Paper SPE 20506 presented at 65th Annual Technical Conference and Exhibition, Sep. 23-26 (1990), Dallas.

Jadhunandan, P.P.,"Effects of Brine Composition, Crude Oil and Aging Conditions on Wettability and Oil Recovery", PhD Thesis, New Mexico Institute of Mining and Technology, New Mexico, 1990.

Jadhunandan, P.P. and Morrow, N.R., "Effect of Wettability on Waterflood Recovery for CrudeOil/Brine/Rock Systems" Paper SPE 22597 presented at 66th Annual Technical Conference and Exhibition, Oct. 6-9 (1991), Dallas.

Jordan, M.M., Sorbie, K.S., Jiang, P., Yuan, M.D., Todd, A.C., "Phosphonate Scale Inhibitor Adsorption/Desorption and the Potential formation Damage in Reconditioned Field Core", Paper SPE 27389 presented at SPE International Symposium on Formation Damage Control, Feb. 7-10 (1994), Lafayette.

Kovscek, A.R., Wong, H. and Radke, C.J., "A PoreLevel Scenario for the Development of Mixed Wettability in Oil Reservoirs", AIChE J., 39, 1072 (1993).

Ma., S., Morrow, N.R., Zhou, X. and Zhang, X. : "Characterisation of Wettability From Spontaneous Imbibition Measurements". Paper Presented at Petroleum Society of CIM 45th Annual Technical Meeting and Aostra 1994 Annual Technical Conference, Calgary, June 12-15.

McDougall, S.R., Dixit, A.B. and Sorbie, K.S., "Network Analogues of Wettability at the Pore Scale", paper Presented at 1995 Developments in Petrophysics Conference, London, Sept. 19-20, Royal Geological Society, London. To be published in Royal Geological Society Special Publication (1996).

Morrow, N.R. "Capillary Pressure Correlations for Uniformly Wetted Porous Media", J. Can. Pet. Tech., 15, 49 (1975).

Salathiel, R.A., " Oil Recovery by Surface Film Drainage in Mixed Wettability Rocks", J. Pet. Tech., Oct., 1216 (1973).

Stauffer, D. and Aharony, A. : "Introduction to Percolation Theory", Revised 2nd Edition, Taylor and Francis, London, 1994.

Wolcott, J.M., Groves (Jr.), F.R., Trujillo, D.E. and Lee, H.G., "Investigation of Crude-oil/Mineral Interactions : Factor Influencing Wettability Alteration ", Paper SPE 21042 presented in International Symposium of Oil Field Chemistry, Feb. 20-22 (1991), Anaheim, California.
Wolcott, J.M., Groves (Jr.), F.R. and Lee, H.G., "Investigation of Crude-oil/Mineral Interactions : Influence of Oil Chemistry on Wettability Alteration ", Paper SPE 25194 presented in International Symposium of Oil Field Chemistry, March 2-5 (1993), New Orleans.

Table 1. Effect of Contact Angle Range Upon Imbibition and Oil Recovery

\begin{tabular}{|c|c|c|}
\hline $\begin{array}{c}\text { Contact } \\
\text { Angle Range }\end{array}$ & $\begin{array}{c}\text { Sw After } \\
\text { Imbibition } \\
\text { Produced } \\
\text { After } \\
\text { Imbibition }\end{array}$ \\
\hline $0-0^{\circ}$ & 0.31 & 26.13 \\
\hline $0-15^{\circ}$ & 0.31 & 26.13 \\
\hline $0-30^{\circ}$ & 0.299 & 25.22 \\
\hline $0-45^{\circ}$ & 0.327 & 28.21 \\
\hline $0-60^{\circ}$ & 0.337 & 29.28 \\
\hline $0-890$ & 0.446 & 40.9 \\
\hline & & \\
\hline $45-890$ & 0.408 & 36.85 \\
\hline $60-890$ & 0.411 & 37.17 \\
\hline
\end{tabular}

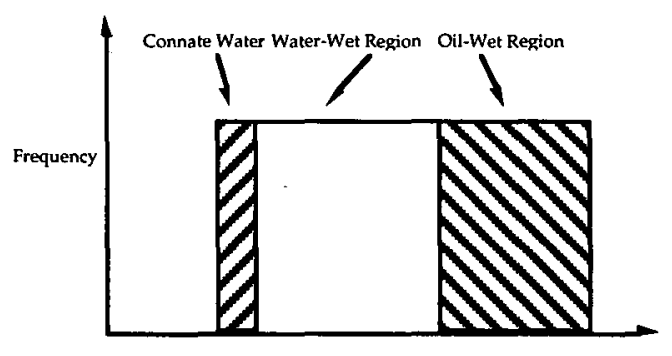

Pore Radius

(a) MIXED-WET SYSTEM

Figure 1. Schematics of mixed-wet pore size distribution 

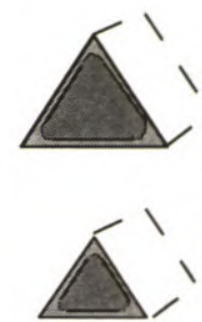

Same Interfacial Curvature at a Given Pc
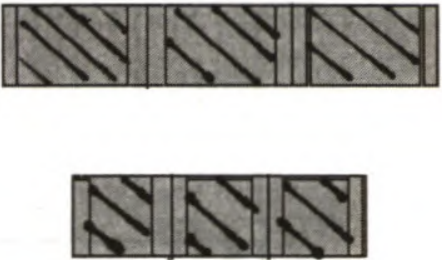

Expanded Pore Surfaces (dark areas remain water-wet, hatched areas are in contact with oil and may become oilwet)

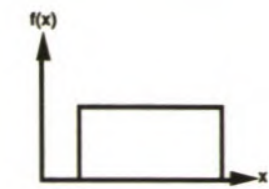

Distribution of Asperity Heights

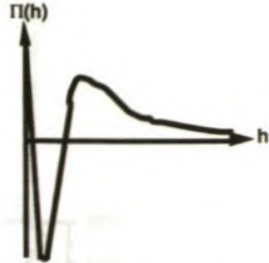

Disjoining Pressure Isotherm

Figure 2. Heuristic argument showing why the largest oil-filled pores may become oil-wet after aging — regardless of pore geometry. Consider two triangular pores of equal length but differing cross-sectional area. Applying thin film theory to this system would result in both pores becoming oil-wet at the same time (critical disjoining pressure is exceeded in both pores simultaneously). However, pore surfaces are rough, so consider a distribution of asperity heights arranged as a regular lattice on the pore surface. As disjoining pressure increases, wetting films rupture at the highest asperities first (largest negative interfacial curvatures here). Hence, problem becomes one of percolation upon the pore wall itself - an oilwet pathway exists across the pore once sufficient asperities have become oil-wet and this will occur first in the widest pore (the thinner pore has a smaller width/length aspect ratio and the percolation problem becomes more and more 1-dimensional as the pore aspect ratio decreases).

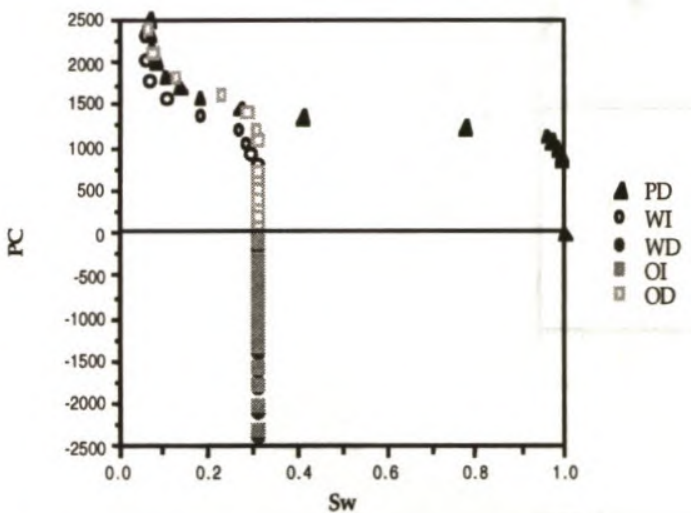

(A)

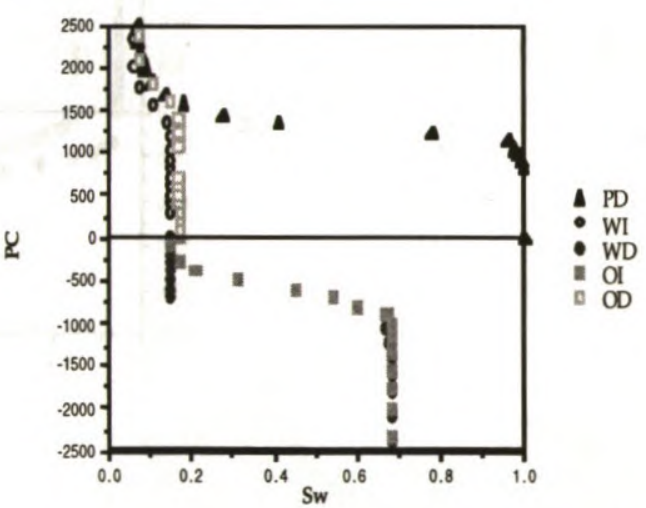

(D)

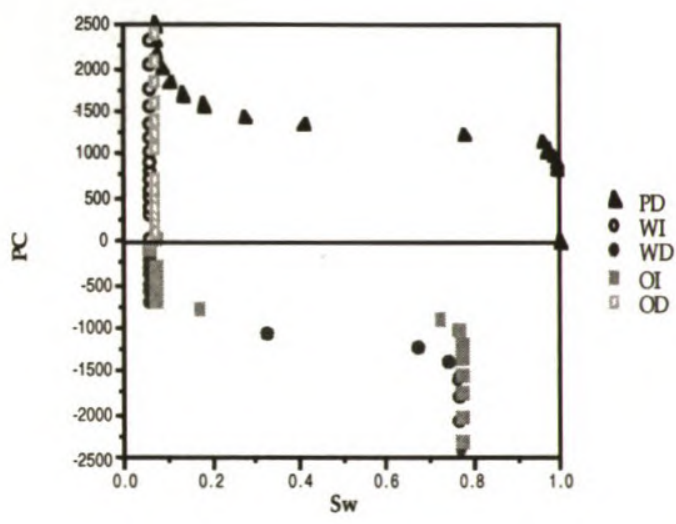

(E)

Figure 3. Capillary pressure curves from five systems with single-valued contact angles: (a) $\alpha=0$; (b) $\alpha=0.469$; (c) $\alpha=0.625$ 


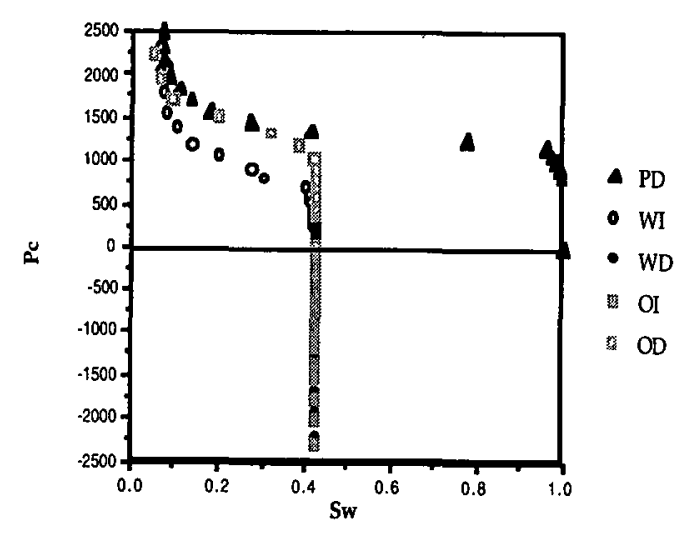

(A)

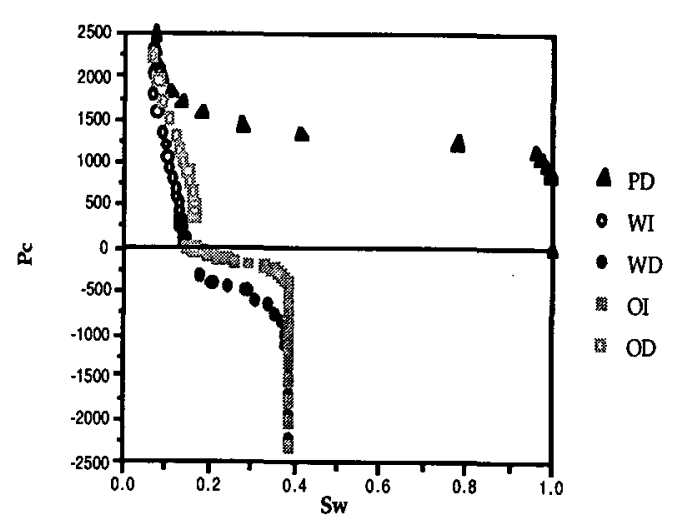

(D)

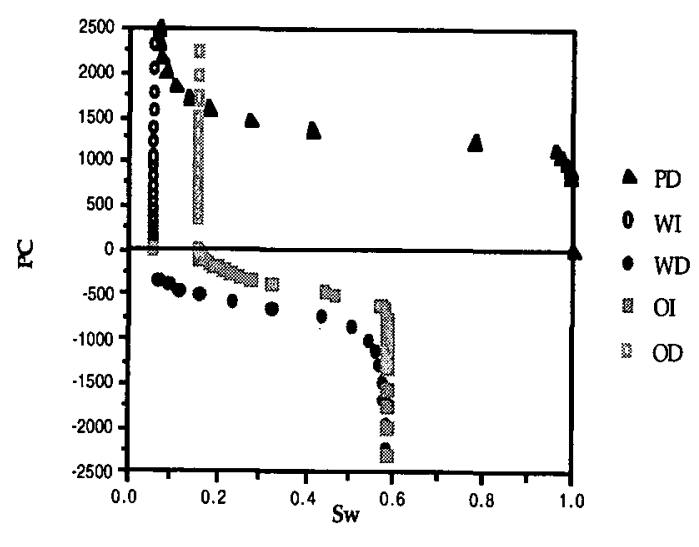

(E) Figure 4. Capillary pressure curves from five systems with distributed contact angles: (a) $\alpha=0$; (b) $\alpha=0.469$; (c)
$\alpha=0.625$. 


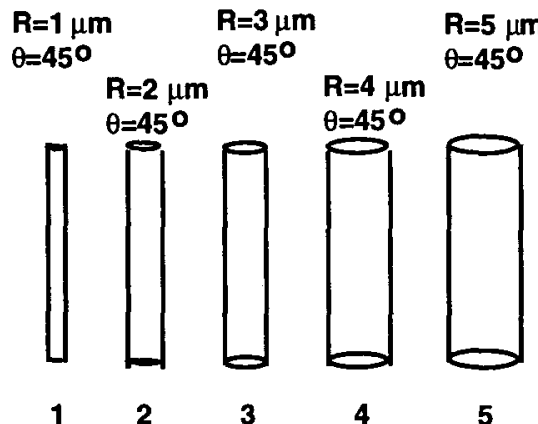

(a)

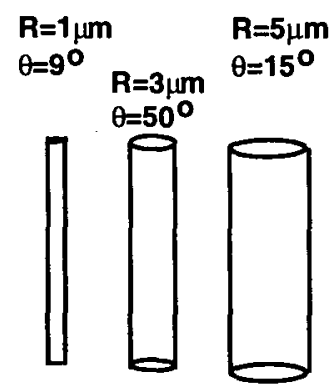

1

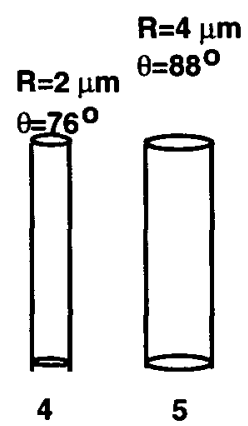

(b)

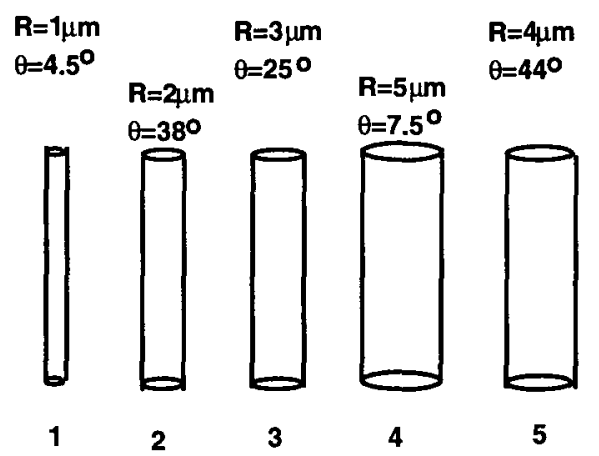

(c)

Figure 5. Tube filling sequences during imbibition for 5 tubes of differing radii: (a) all contact angles $=45^{\circ}$; (b) contact angles distributed from $0-90^{\circ}$; (c) contact angles half those in (B).

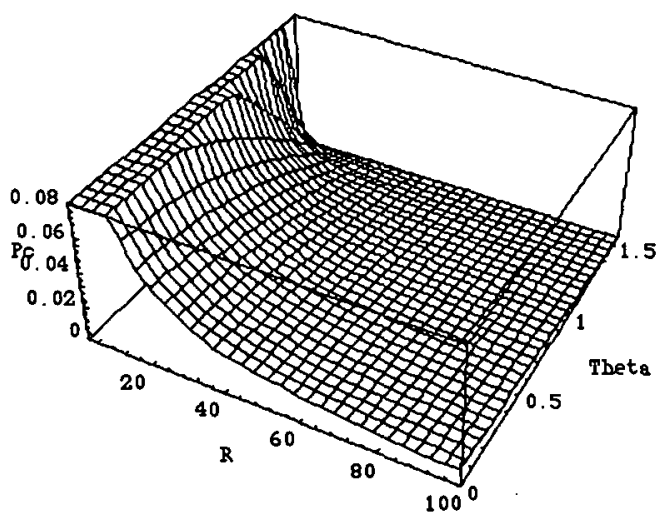

(a)

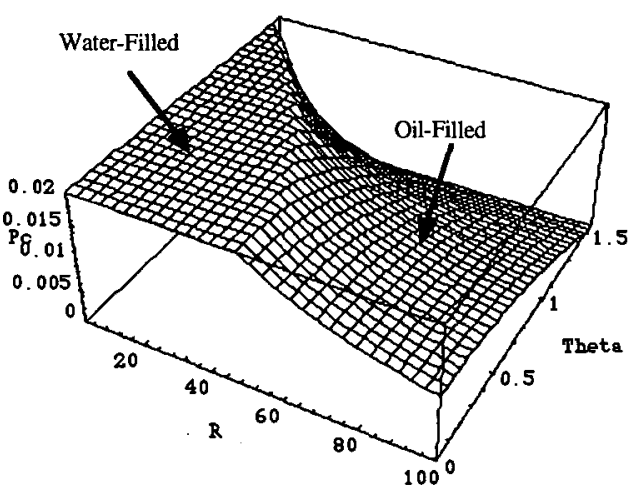

(b)

Figure 6. (a) Schematic example of a capillarity surface, $P_{c}(r, \theta)$ (theta in radians); (b) water and oil pore occupancy for imbibition 


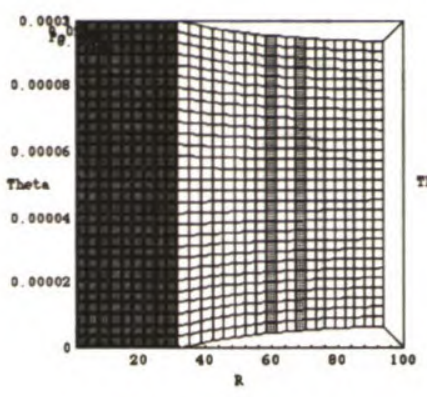

(a)

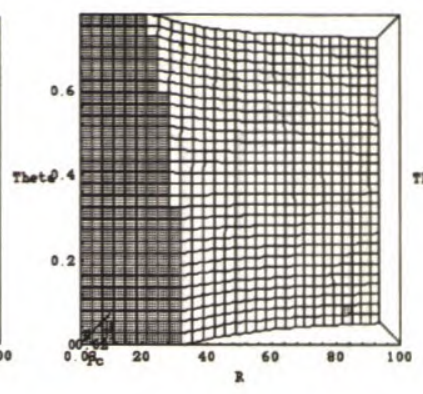

(b)

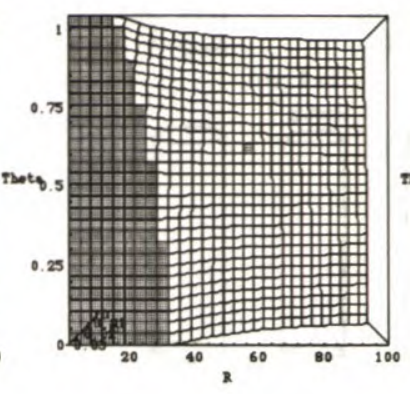

(c)

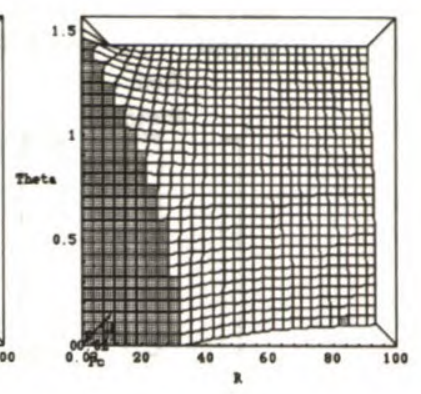

(d)

Figure 7. Capillarity surfaces, $P_{c}(r, \theta)$, for various ranges of water-wet contact angle; the water-filled pores are shown shaded for each contact angle range (a) $\theta=0^{\circ}$, (b) $\theta=0-45^{\circ}$, (c) $\theta=0-60^{\circ}$ (d) $\theta=0-90^{\circ}$.

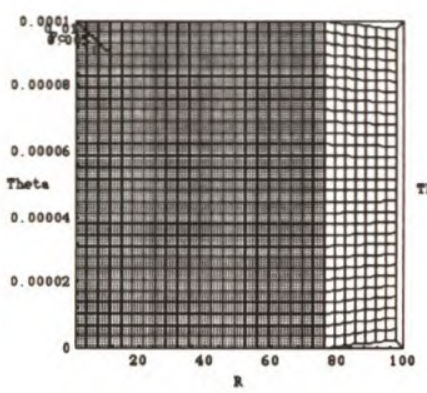

(a)

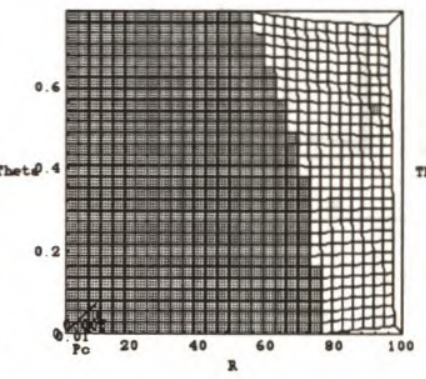

(b)

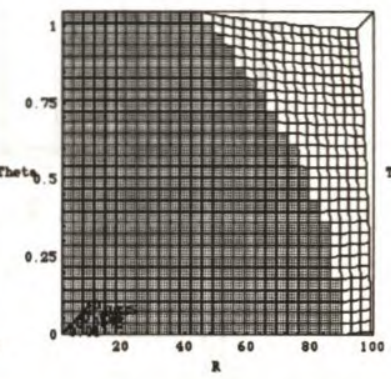

(c)

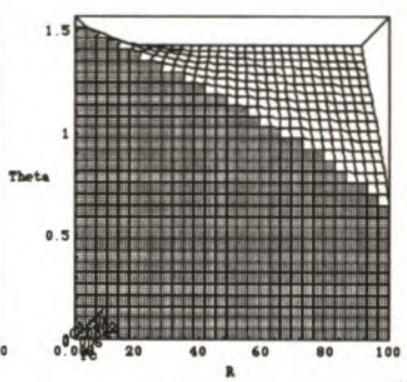

(d)

Figure 8. Distribution of water and oil filled pores at residual oil; the water-filled pores are shown shaded for each contact angle range; (a) $\theta=0^{\circ}$, (b) $\theta=0-45^{\circ}$, (c) $\theta=0-60^{\circ}$ (d) $\theta=0-90^{\circ}$.

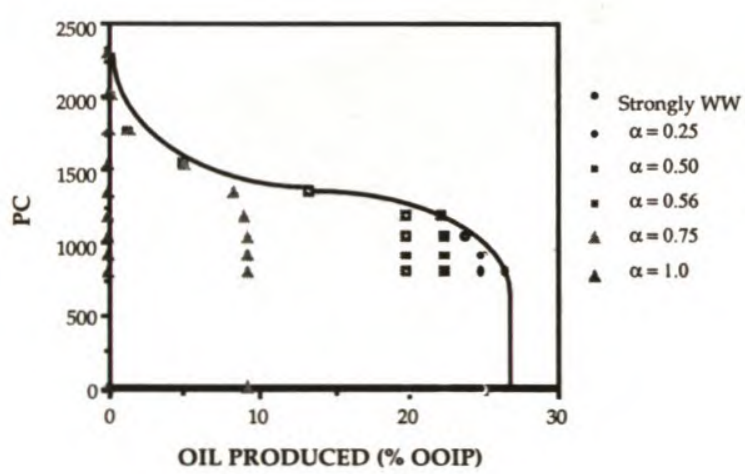

(a)

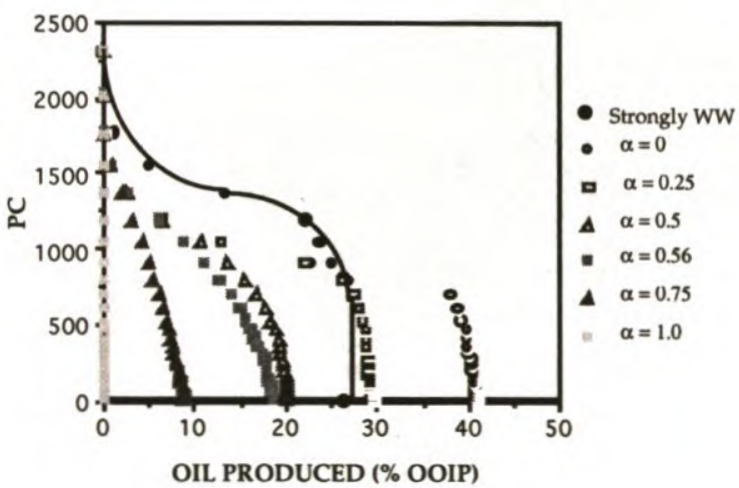

(b)

Figure 9 Spontaneous water imbibition curves from mixed-wet systems; (a) single valued contact angles (b) distributed contact angles. NB the values of $\alpha$ shown here actually correspond to the fraction of oil-filled pores that become oil-wet after primary drainage. To obtain the fraction of total pores that are oil-wet, multiply these values by 0.625 . 